\title{
Optimal Large-scale Storage Placement in Single Generator Single Load Networks
}

\author{
Christos Thrampoulidis, Subhonmesh Bose, Babak Hassibi \\ California Institute of Technology.
}

\begin{abstract}
Large-scale storage will play an increasingly important role in future power grids. As a result, how to optimally place storage in such networks, is an important investment problem. Furthermore, since the allocation of storage resources is static, i.e., it is not feasible to move storage around in a dynamic fashion, it is important to derive optimal such allocations that are robust to the values of the load profiles and other network parameters, such as the line flow constraints. For a single generator single load network, and for a cost of generation that is quadratic in the generation power, we show that, for any given amount of storage resources, placing storage at the demand node is always optimal. This result is true regardless of the demand profile and flow constraints, and therefore is robust. As a byproduct of this result, for a fixed demand profile, we characterize the dependence of the optimal production cost on the flow constraints and on the available storage resources.
\end{abstract}

Index Terms-Electricity storage, load shifting, storage placement.

\section{INTRODUCTION}

Energy storage can potentially be used for various services to enhance sustainability, reliability, efficiency, and better asset utilization of the power grid, e.g, [1]-[5]. Recent advances in large scale storage technologies coupled with the advent of intermittent renewable generation has spurred a lot of research interest in this area [4], [6]-[8]. Applications of energy storage can be classified according to the time scale of operation [3], [9]. At fast-time scales (seconds to minutes), storage is mainly used to mitigate variability of renewable generation and demand and reduce the role of ancillary services to balance demand and supply, e.g., [10]-[12]. On a slower time scale (over hours), bulk storage devices aims at load shifting, i.e., generate only when it is cheap and supply the variation in load by charging and discharging storage devices accordingly [4], [9].

Several authors have investigated the optimal control policy for the storage unit. Koutsopoulos et al. [13] and $\mathrm{Su}$ et al. [12] examine the operation of a single storage device, thus neglecting the network constraints. On the other hand, Kanoria et al. in [14], Gayme et al. in [15] and Chandy et al. in [16] explicitly model the role of the networks in the operation of distributed storage resources. The engineering constraints are

Emails: (cthrampo, boses, hassibi) @caltech.edu. This work was supported in part by NSF under grants CCF-0729203, CNS0932428 and CCF-1018927, NetSE grant CNS 0911041, Office of Naval Research MURI grant N00014-08-1-0747, Caltech Lee Center for Adv. Net., ARPA-E grant DE-AR0000226, Southern Cali. Edison, Nat. Sc. Council of Taiwan, R.O.C. grant NSC 101-3113-P-008-001, Resnick Inst., Okawa Foundation and Andreas Mentzelopoulos Scholarships for the Univ. of Patras. designed based on one of the two popular optimal power flow (OPF) models, namely, AC-OPF [17] and DC-OPF [18], [19]. Storage resources are assumed to be known a priori in these settings.

As storage technologies are improving, the optimal sizing of these resources in a grid is gaining importance. In [20] and [21], the authors consider this question from a purely economic point of view. Capacity provisioning of energy resources with network constraints of the physical system has been addressed, e.g., [12], [14]. Optimal placement and sizing storage units have been studied in [22], [23] using simulations. While [14], [22] rely on the DC-OPF model, [23] uses a convex relaxation of AC-OPF based on [24], [25].

In this paper, we address the optimal storage placement problem for slow time scales to reduce the cost of generation on a single generator single load system with a finite line capacity. With any available storage budget, we prove that it is always optimal to place all the capacity at the demand node, regardless of the line capacity or demand profile when minimizing a quadratic generation cost. This is an investment decision problem but we also solve the optimal control of the storage devices as a by-product of our analysis. To the best of our knowledge, our work is the first to derive analytic results for the placement problem. Furthermore, we characterize the dependence of the optimal production cost on the line flow capacity and the available storage budget.

The paper is organized as follows. We formulate the optimal storage placement problem in Section II and derive the analytic results in Section III. The results are illustrated through examples in Section IV. We conclude in Section V with directions for future work.

\section{Problem Formulation}

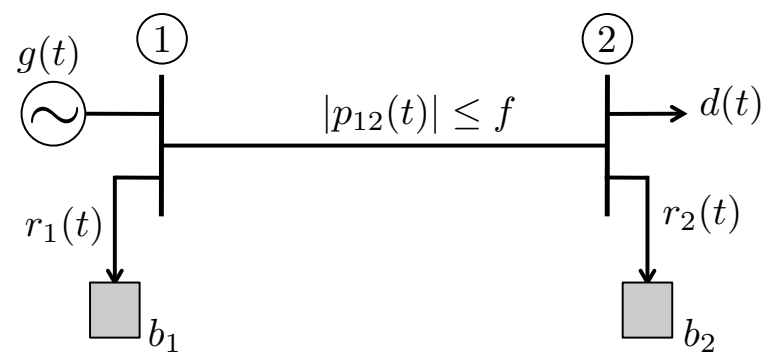

Fig. 1: Single generator single load network with storage at each node. The total available storage budget is $h=b_{1}+b_{2}$. 
Consider a single generator single load network as shown in Figure 1. Generator at bus 1 is connected to a load (or demand) at bus 2 using a single line. We adopt the $D C$ power flow model [18] for our network and thus reactive power flows and power losses on the line are neglected.

In our model, time is discrete and is indexed by $t$. We use the following notation.

- $d(t)$ is the real power demand at the load bus, at time $t$, which is assumed to be known.

- $g(t)$ is the real power generation at the generator bus at time $t$ and it satisfies

$$
0 \leq g(t) \leq \bar{g}
$$

where $\bar{g}$ is the generation capacity.

- $c(g(t))$ is the cost of generating $g(t)$ at time $t$. Note that the production cost is independent of time. Also, we use a quadratic cost function [17]:

$$
c(g(t))=\frac{1}{2}[g(t)]^{2} .
$$

- $p_{12}(t)$ is the real power flow from the generator bus to the load bus at time $t$. This is limited by the line-flow capacity $f$ due to thermal and stability considerations as follows:

$$
\left|p_{12}(t)\right| \leq f .
$$

- $r_{k}(t)$ is the average power pumped into the storage unit at buses $k=1,2$ at time $t$. The energy transacted over a time-step is converted to power units by dividing it by the length of the time-step [23]. This transformation conveniently allows us to formulate the problem in units of power. Notice that $r_{k}(t)$ can be positive or negative depending on whether power flows in or out of the storage device. For practical storage devices, the roundtrip efficiency is typically less than one. For the purposes of this paper, however, we neglect these inefficiencies. $b_{k} \geq 0$ is the storage capacity at bus $k$ and $b_{k}^{0}$ is the storage level at node $k$ at time $t=0$. Thus $r_{k}(t)$ for all $t$ satisfies

$$
0 \leq b_{k}^{0}+\sum_{\tau=1}^{t} r_{k}(\tau) \leq b_{k} .
$$

- $h$ is the total available storage budget that can be installed on the network. The algorithm solves for how much of this available capacity to install at each node. Thus we have

$$
b_{1}+b_{2} \leq h .
$$

To maintain appropriate power balance at each bus of the network we require, for all $t$,

$$
g(t)-r_{1}(t)=p_{12}(t)=d(t)+r_{2}(t) .
$$

Demand profiles often show diurnal variations, i.e., they exhibit cyclic behavior. Let $T$ time steps be the common cycle length of this variation. In particular, for all $t \geq 0$, assume

$$
d(t+T)=d(t) .
$$

Optimally placing storage over an infinite horizon is then equivalent to solving this problem over a single cycle, provided the state of the system at the end of a cycle is the same as its initial condition, i.e., the levels of storage at the beginning and end of each cycle are equal [23]. Thus for $k=1,2$, we have

$$
\sum_{t=1}^{T} r_{k}(t)=0 .
$$

We also assume $b_{1}^{0}=b_{2}^{0}=0$, so that the storage units are empty at the time of installation.

Using the above notation, we define the following optimization problems.

Storage placement problem $P$ :

$$
\begin{aligned}
\underset{g(t), r_{1}(t), b_{1}, r_{2}(t), b_{2}}{\operatorname{minimize}} & \sum_{t=1}^{T} c(g(t)) \\
\text { subject to } & (1),(3),(4),(5),(6),(7) .
\end{aligned}
$$

\section{Restricted storage placement problem $\Pi$ :}

$$
\begin{aligned}
\underset{g(t), r_{1}(t), b_{1}, r_{2}(t), b_{2}}{\operatorname{minimize}} & \sum_{t=1}^{T} c(g(t)) \\
\text { subject to } & (1),(3),(4),(5),(6),(7), \\
& b_{1}=0 .
\end{aligned}
$$

Problem $\Pi$ corresponds to placing no storage at the generator bus (bus 1 in Figure 1). We relate the problems $P$ and $\Pi$ in the next section.

\section{RESUltS}

Let $p^{*}$ and $\pi^{*}$ be the optimal cost for the problems $P$ and $\Pi$ respectively. Using this notation we present the main result of this paper.

Theorem 1: If $P$ is feasible, then $\Pi$ is feasible and $p^{*}=\pi^{*}$. Theorem 1 states that, for any available storage budget, placing all the available storage at the load bus is always optimal, regardless of the demand pattern, the time-invariant capacity of generation and the line-flow capacity. In our model, the demand profile is deterministic, however, Theorem 1 holds for arbitrary demand profiles and hence applies to the stochastic case. Our proof technique generalizes to a network where the load bus (bus 2 in Figure 1) is replaced with a network of loads (see [26]), but, we only present the proofs for the simple 2bus system shown in Figure 1. Thus, our result holds for most distribution networks [27] and isolated transmission networks, e.g., power network in Catalina island [28].

Proof: For any variable $z$ in problem $P$, let $z^{*}$ be the value of the corresponding variable at the optimum. We use the following result, which we prove in the appendix.

Lemma 2: If $P$ is feasible, then $g^{*}(t) \leq f$ for all $t=$ $1,2, \ldots T$.

Note that for each $1 \leq t \leq T, g(t)+r_{1}(t)$ is the amount of power that flows from bus 1 to bus 2 and hence $g(t)+$ $r_{1}(t) \leq f$. From lemma 2 it follows that at the optimum, $g^{*}(t), t=1,2, \ldots T$ itself defines a feasible flow over this line. 
We use this to construct an optimum of $\Pi$ using an optimum of $P$. In particular, define

$$
\begin{gathered}
g^{\prime}(t)=g^{*}(t), \quad r_{1}^{\prime}(t)=0, \quad b_{1}^{\prime}=0, \\
r_{2}^{\prime}(t)=r_{1}^{*}(t)+r_{2}^{*}(t), \quad b_{2}^{\prime}=b_{1}^{*}+b_{2}^{*} .
\end{gathered}
$$

Using lemma 2, it can be checked that $\left(g^{\prime}(t), r_{1}^{\prime}(t), r_{2}^{\prime}(t), b_{1}^{\prime}, b_{2}^{\prime}\right)$ is a feasible point of $\Pi$ with cost $p^{*}$. Since $\pi^{*} \geq p^{*}$, it follows that $\left(g^{\prime}(t), r_{1}^{\prime}(t), r_{2}^{\prime}(t), b_{1}^{\prime}, b_{2}^{\prime}\right)$ is optimal for $\Pi$. This completes the proof of Theorem 1 .

In the problems $P$ and $\Pi$, we solve for the optimal placement and control of storage in a power-network, given the demand profile $d(t), t=1,2, \ldots, T$, generation capacity $\bar{g}$, storage budget $h$ and the flow limit $f$ on the line joining buses 1 and 2. Now we analyze the placement problem by suitably changing $f$ and $h$. To explicitly state this parameterization, we use the following notation. Let $P(f, h)$ denote the storage placement problem and $p^{*}(f, h)$ be its optimal cost respectively. Using this notation we present the following observations. For the proofs, see [26]. For the purpose of the ensuing analysis, assume henceforth that there is no limit on the available generation capacity, i.e., $\bar{g}=+\infty$. See [26] for the general case.

Proposition 3: For any $h$, problem $P(f, h)$ is feasible iff $f \geq f_{\min }$, and, $p^{*}(f, h)=p^{*}\left(f_{\min }, h\right)$ for all $f \geq f_{\min }$, where

$$
\begin{aligned}
& f_{\min }=\max \left\{\max _{1 \leq t \leq T}\left(\frac{\sum_{i=1}^{t} d(i)}{t}\right),\right. \\
&\left.\max _{1 \leq t_{1}<t_{2} \leq T}\left(\frac{\sum_{i=t_{1}+1}^{t_{2}} d(i)-h}{t_{2}-t_{1}}\right)\right\} .
\end{aligned}
$$

We interpret this result as follows. Suppose demand profile $d(t), t=1,2, \ldots T$ and total storage budget $h$ are fixed. Then $f_{\text {min }}$ can be calculated from $d(t)$ and $h$ from (8). For line flow limits $f<f_{\min }$, the problem $P(f, h)$ is infeasible, i.e., the loads cannot be satisfied by the network. Notice that $f_{\min }$ for $h>0$ is less than $f_{\min }$ for $h=0$. Thus, storage can be used to reduce cost of operation avoiding transmission upgrades [21]. Interestingly, for $f \geq f_{\min }$, the optimal cost of operation does not depend on $f$. From transmission or distribution planning perspective, investment in line capacities over $f_{\min }$ does not reduce the cost of operation.

We provide some intuition behind the two terms in equation (8). At each time $t$, the net difference between generation and demand till time $t$ equals the power stored in storage devices up to that time, which is non-negative. Using this, we can bound $\max _{1 \leq t \leq T} g^{*}(t)$ from below by the first term in (8). This translates to a bound on $f$ using lemma 2 . For the second term, we derive a similar bound on $\max _{1 \leq t \leq T} g^{*}(t)$ using the fact that the power extracted from storage devices over any interval cannot exceed the total storage budget $h$.

Now, we characterize the behavior of $P(f, h)$ and its optimal cost $p^{*}(f, h)$ as a function of $h$.

Proposition 4: Suppose $f \geq \max _{1 \leq t \leq T}\left(\frac{\sum_{i=1}^{t} d(i)}{t}\right)$. Then $P(f, h)$ is feasible iff $h \geq h_{\min }$, and, $p^{*}(f, h)$ is convex and non-increasing in $h$, where

$$
h_{\text {min }}=\max \left\{\max _{1 \leq t_{1} \leq t_{2} \leq T}\left[-\sum_{i=t_{1}}^{t_{2}}(f-d(i))\right], \quad 0\right\} .
$$

The condition $f \geq \max _{1 \leq t \leq T}\left(\frac{\sum_{i=1}^{t} d(i)}{t}\right)$ implies that there is some large $h>0$ for which $P(f, h)$ is feasible. If this condition is violated, the problem remains infeasible no matter how large the storage budget $h$ is. Given the line flow limit $f$ and the demand profile $d(t), t=1,2, \ldots T$, the minimum storage required to serve the demand is given by $h_{\min }$ as in (9). Also, more the storage budget, lesser is the generation cost and hence $p^{*}(f, h)$ is decreasing in $h$. The convexity, however, implies that there is diminishing marginal returns on the investment on storage, i.e., the benefit of the first unit installed is more than that from the second unit.

It is easy to observe that as we allow larger storage budget, the generation cost does not reduce beyond a point, i.e., there exists $h_{\text {sat }}$ such that $p^{*}(f, h)=p^{*}\left(f, h_{\text {sat }}\right)$ for all $h \geq h_{\text {sat }}$. We define some notation and then calculate $h_{\text {sat }}$ in Proposition 5. Construct the sequence $\left\{\tau_{n}\right\}_{n=0}^{N}$ as follows. Let $\tau_{0}=0$. Define $\tau_{n}$ iteratively:

$$
\tau_{n}=\underset{\tau_{n-1}+1 \leq t \leq T}{\arg \max }\left(\frac{\sum_{i=\tau_{n-1}+1}^{t} d(i)}{t-\tau_{n-1}}\right),
$$

for $1 \leq n \leq N$, where $N$ is the smallest integer for which $\tau_{N}=T$. Note that the sequence depends only on the demand profile $d(t), t=1,2, \ldots T$.

Proposition 5: Suppose $f \geq \max _{1 \leq t \leq T}\left(\frac{\sum_{i=1}^{t} d(i)}{t}\right)$. Then

$$
\begin{aligned}
h_{s a t}=\max _{1 \leq n \leq N}\left[\max _{\tau_{n-1}+1 \leq t \leq \tau_{n}}\right. & \left\{\left(\sum_{i=\tau_{n-1}+1}^{\tau_{n}} d(i)\right) \frac{t-\tau_{n-1}}{\tau_{n}-\tau_{n-1}}\right. \\
& \left.\left.-\left(\sum_{i=\tau_{n-1}+1}^{t} d(i)\right)\right\}\right] . \quad \text { (11) }
\end{aligned}
$$

Notice, that $h_{\text {sat }}$ is a function of only the demand profile and is independent of the line flow capacity.

\section{EXAMPLES}

We illustrate propositions 3, 4 and 5 through an example. All units are in per units (p.u.). Consider an hourly load profile as shown in Figure 2. The optimal generation profile $g^{*}(t)$ for $P$ (or equivalently $\Pi$ ) has been plotted for a storage budget $h=1$ and line flow capacity $f=0.85$. In figure 2 , $\max _{t} g^{*}(t) \leq f$ as in lemma 2 and hence from theorem 1, we have $p^{*}=\pi^{*}$.

Consider the plots in figure 3. First we plot $p^{*}(f=0.85, h)$ for $h$ in $[0,3]$ in figure $3 a$. Notice that $f \leq \max _{t} d(t)$, i.e., the problem is infeasible in the absence of storage. We calculate $h_{\min }=0.226$ and $h_{\text {sat }}=2.598$ from propositions 4 and 5 respectively. In figure $3 \mathrm{~b}$, we plot $p^{*}(f, h=1)$ for $f$ in $[0,2]$. As in proposition 3, the problem is infeasible for $f<f_{\min }=$ 0.683 and the optimal cost does not change for $f \geq f_{\min }$. 


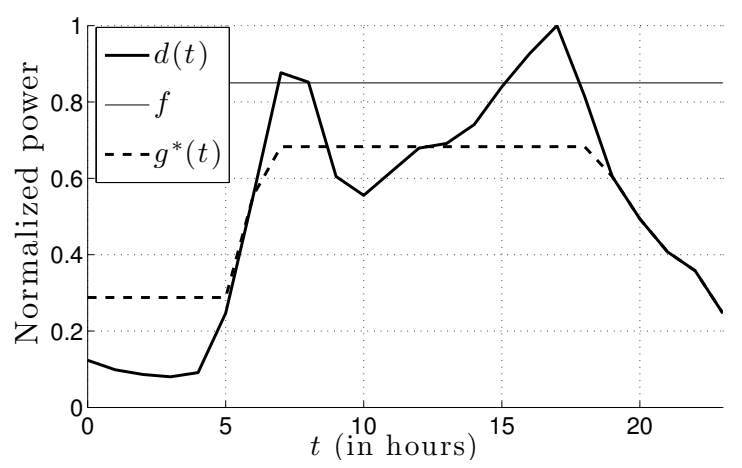

Fig. 2: Typical hourly load profile and optimal generation portofolio for line flow capacity $f=0.85$ and storage budget $h=1$.

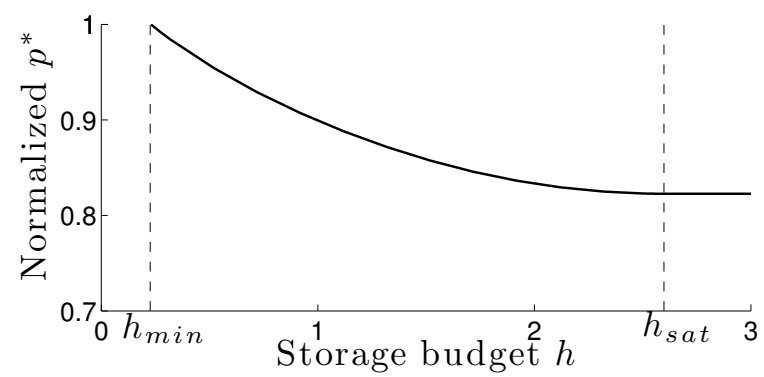

(a) $p^{*}(f=0.85, h)$

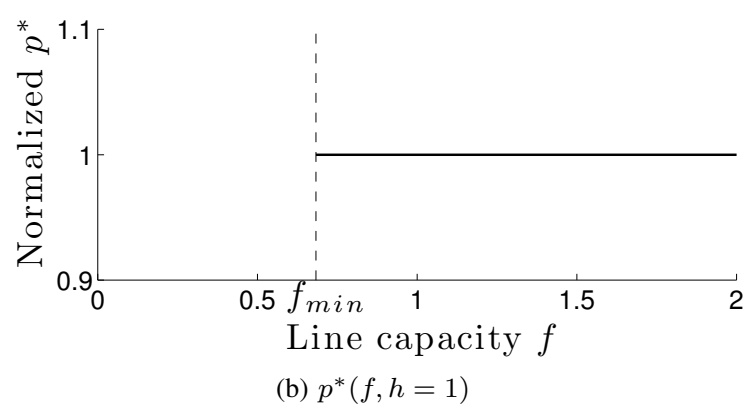

Fig. 3: $p^{*}(f, h)$ and illustration of $h_{m i n}, h_{s a t}$ and $f_{\text {min }}$

\section{CONCLUSION}

In this paper, we have considered the problem of optimal placement of storage in a single generator single load power network. The main result (theorem 1) states that for any available storage budget, it is always optimal to put all the storage at the load bus, irrespective of demand profile, generation capacity and line-flow limit. The result holds more generally and is a subject of future work [26]. With more grid-level storage options like compressed air energy storage [9] available, this problem is gaining importance for the transmission and distribution system planners.

In addition to the storage placement problem, we have obtained the minimum line-flow limit required under some available storage budget in proposition 3. Furthermore, we have analyzed the cost as a function of the available storage budget and derived closed-form expressions for the minimum and maximum storage budget required when line flow capacities are fixed in propositions 4 and 5 respectively.

A possible direction of future work is to incorporate intermittent sources of generation and stochastic demands and also make the storage model to incorporate losses. Application of such results to more networks and stochastic settings is part of our ongoing research [26].

\section{ACKNOWLEDGEMENTS}

The authors gratefully acknowledge Prof. K. Mani Chandy at California Institute of Technology and Mr. Paul DeMartini from Resnick Institute for their helpful comments.

\section{APPENDIX}

We prove lemma 2 in this section. The result is restated. If $P$ is feasible, then $g^{*}(t) \leq f$ for all $t=1,2, \ldots T$.

Proof: We start with some notation. For any vector or matrix $v$, let $v^{\dagger}$ denote the transpose of $v$. For any variable $z(t)$ that depends on time $t$, let $z$ denote the $T \times 1$ vector $(z(1), z(2), \ldots, z(T))^{\dagger}$. For any two vectors $v_{1}$ and $v_{2}$, we say $v_{1} \geq v_{2}$ iff $v_{1}-v_{2}$ is elementwise non-negative. Let $\mathbf{0}$ and $\mathbf{1}$ respectively denote vectors of all zeros and ones of appropriate size. Define the $T \times T$ matrix $J$ as the lower triangular part of $1 \mathbf{1}^{\dagger}$. Using this notation, $P$ can be written as follows.

$$
\begin{aligned}
\underset{g, r_{1}, r_{2}, b_{1}, b_{2}}{\operatorname{minimize}} & \frac{1}{2} g^{\dagger} g \\
\text { subject to } & p_{12}=g-r_{1}, \\
& p_{12}=d+r_{2}, \\
& -f \mathbf{1} \leq p_{12} \leq f \mathbf{1}, \\
& \mathbf{0} \leq J r_{k} \leq b_{k} \mathbf{1}, \quad k=1,2, \\
& \mathbf{1}^{\dagger} r_{k}=0, \quad k=1,2, \\
& g \geq \mathbf{0}, \\
& g \leq \bar{g} \mathbf{1}, \\
& b_{1}+b_{2} \leq h .
\end{aligned}
$$

Assume $P$ is feasible throughout. If $g=\mathbf{0}$ is feasible for $P$, then the result follows trivially. Assume henceforth $\max _{1 \leq t \leq T} g(t)>0$, for all feasible solutions.

If $\bar{g} \leq f$, then lemma 2 clearly holds. If not, we first consider the case where $\bar{g}=+\infty$ and then use it to prove the claim for a finite $\bar{g}>f$.

Let the Lagrange multipliers for equations (12a) and (12f) be the $T \times 1$ vectors $\mu$ and $\lambda$ respectively. For the upper and lower inequalities in (12d) let the Lagrange multipliers be $u_{k}$ and $\ell_{k}$ respectively for $k=1,2$. Also let $\nu_{k}$ be the Lagrange multipliers for (12e) for $k=1,2$.

$P$ is a quadratic program with linear constraints that is bounded below and Slater's condition holds for $P$. Thus $P$ and its dual have a finite primal-dual optimal point that satisfies the Karush-Kuhn-Tucker (KKT) conditions and the duality gap is zero [29]. We analyze this optimal point in more detail. The KKT conditions include primal feasibility, dual feasibility and 
complementary slackness. We only present a subset of these conditions:

$$
\begin{array}{r}
g^{*}-\mu^{*}-\lambda^{*}=\mathbf{0}, \\
\mu^{*}-J^{\dagger} \ell_{1}^{*}+J^{\dagger} u_{1}^{*}+\nu_{1}^{*} \mathbf{1}=\mathbf{0}, \\
\ell_{1}^{*} \geq \mathbf{0}, \quad u_{1}^{*} \geq \mathbf{0}, \\
\left(\ell_{1}^{*}\right)^{\dagger} J r_{1}^{*}=0, \quad\left(u_{1}^{*}\right)^{\dagger} J r_{1}^{*}=b_{1}^{*}\left(u_{1}^{*}\right)^{\dagger} \mathbf{1}, \\
\lambda^{*} \geq \mathbf{0}, \quad\left(\lambda^{*}\right)^{\dagger} g^{*}=0 .
\end{array}
$$

From (13a), (13e) and (12f), we have $g^{*}(t)=\max \left\{\mu^{*}(t), 0\right\}$. Also, since $g^{*} \neq \mathbf{0}$, we have

$$
\max _{1 \leq t \leq T} g^{*}(t)=\max _{1 \leq t \leq T} \mu^{*}(t)>0 .
$$

Multiplying equation (13b) by $\left(J^{\dagger}\right)^{-1}$, it follows that

$$
\left(J^{\dagger}\right)^{-1} \mu^{*}=\ell_{1}^{*}-u_{1}^{*}-\nu_{1}^{*} e_{T} .
$$

where the $T \times 1$ vector $e_{T}$ is defined as $(0,0, \cdots, 0,1)^{\dagger}$. This relation is well defined since $J^{\dagger}$ is invertible. Simplifying the above result, we get:

$$
\mu^{*}(t)-\mu^{*}(t+1)=\ell_{1}^{*}(t)-u_{1}^{*}(t), \quad t=1,2, \ldots, T-1,
$$

$$
\text { and } \mu^{*}(T)=\ell_{1}^{*}(T)-u_{1}^{*}(T)-\nu_{1} .
$$

Define

$$
t_{\max }:=\max \left\{t \mid \mu^{*}(t)=\max _{1 \leq t^{\prime} \leq T} \mu^{*}\left(t^{\prime}\right) \text { and } 1 \leq t \leq T\right\} .
$$

We now show that $\sum_{t=1}^{t_{\max }} r_{1}^{*}(t)=0$. If $t_{\max }=T$, this follows from (12e). If $t_{\max }<T$, then from equations (15) and (16), we have

$$
\mu^{*}\left(t_{\max }\right)-\mu^{*}\left(t_{\max }+1\right)=\ell_{1}^{*}\left(t_{\max }\right)-u_{1}^{*}\left(t_{\max }\right)>0,
$$

and hence $\ell_{1}^{*}\left(t_{\max }\right)>0$ and $u_{1}^{*}\left(t_{\max }\right)=0$. From complementary slackness condition in (13d), it follows that $\sum_{t=1}^{t_{\max }} r_{1}^{*}(t)=$ 0 . Combining equations (14) and (16), we have

$$
\begin{aligned}
\max _{1 \leq t \leq T} g^{*}(t) & =\mu^{*}\left(t_{\max }\right) \\
& =p_{12}^{*}\left(t_{\max }\right)+r_{1}^{*}\left(t_{\max }\right) \\
& =\underbrace{p_{12}^{*}\left(t_{\max }\right)}_{\leq f}+\underbrace{\sum_{\max }^{t_{t=1}} r_{1}^{*}(t)}_{=0}-\underbrace{\sum_{t=1}^{t_{\max }-1} r_{1}^{*}(t)}_{\geq 0 \text { from }(12 \mathrm{~d})} \\
& \leq f .
\end{aligned}
$$

Thus, $g^{*} \leq f \mathbf{1}$, for the case $\bar{g}=+\infty$. For a finite $\bar{g}>f$, note that the above $g^{*}$ is feasible and hence optimal for $P$. This completes the proof.

Remark $\mu^{*}(t)$ (and hence $g^{*}(t)$ ) is piecewise constant and the discontinuities occur at times where the storage level at bus 1 is either full or empty. See [26] for details.

\section{REFERENCES}

[1] R. Schainker, "Executive overview: Energy storage options for a sustainable energy future," in Proc. of IEEE PES General Meeting, 2004, pp. 2309-2314.

[2] A. Nourai, "Installation of the first distributed energy storage system (dess) at american electric power (aep)," Sandia Nat. Labs, 2007.

[3] P. Denholm, E. Ela, B. Kirby, and M. Milligan, "The role of energy storage with renewable electricity generation," 2010.

[4] D. Rastler, Electricity Energy Storage Technology Options: A White Paper Primer on Applications, Costs and Benefits. Electric Power Research Institute, 2010.

[5] P. Varaiya, F. Wu, and J. Bialek, "Smart operation of smart grid: Risklimiting dispatch," Proc. of the IEEE, vol. 99, no. 1, pp. 40-57, 2011.

[6] J. Greenberger. (2011, Nov.) The smart grid's problem may be storage's opportunity. [Online]. Available: http://theenergycollective.com/jimgreenberger/70813/smart-grids-problem-may-be-storages-opportunity

[7] D. Lindley, "Smart grids: The energy storage problem." Nature, vol. 463 , no. 7277 , p. $18,2010$.

[8] S. Chu and A. Majumdar, "Opportunities and challenges for a sustainable energy future," Nature, vol. 488, no. 7411, pp. 294-303, 2012.

[9] J. Eyer and G. Corey, "Energy storage for the electricity grid: Benefits and market potential assessment guide," Sandia Nat. Lab., 2010.

[10] H. Oh, "Optimal planning to include storage devices in power systems," Pow. Sys., IEEE Trans. on, vol. 26, no. 3, pp. 1118-1128, 2011.

[11] Y. M. Atwa and E. F. El-Saadany, "Optimal allocation of ESS in distribution systems with a high penetration of wind energy," IEEE Trans. on Power Sys., vol. 25, no. 4, pp. 1815-1822, Nov. 2010.

[12] H. Su and A. Gamal, "Modeling and analysis of the role of fast-response energy storage in the smart grid," arXiv preprint arXiv:1109.3841, 2011.

[13] I. Koutsopoulos, V. Hatzi, and L. Tassiulas, "Optimal energy storage control policies for the smart power grid," in Smart Grid Communications (SmartGridComm), 2011 IEEE International Conference on. IEEE, 2011, pp. 475-480.

[14] Y. Kanoria, A. Montanari, D. Tse, and B. Zhang, "Distributed storage for intermittent energy sources: Control design and performance limits," in Communication, Control, and Computing (Allerton), 2011 49th Annual Allerton Conference on. IEEE, 2011, pp. 1310-1317.

[15] D. Gayme and U. Topcu, "Optimal power flow with large-scale energy storage integration," IEEE Trans. on Power Sys., to appear, 2012.

[16] M. Chandy, S. Low, U. Topcu, and H. Xu, "A simple optimal power flow model with energy storage," in Proc. of Conf. on Decision and Ctrl., 2010.

[17] A. R. Bergen and V. Vittal, Power Systems Analysis, 2nd ed. Prentice Hall, 2000.

[18] K. Purchala, L. Meeus, D. Van Dommelen, and R. Belmans, "Usefulness of dc power flow for active power flow analysis," in Power Engineering Society General Meeting, 2005. IEEE. IEEE, 2005, pp. 454-459.

[19] K. S. Pandya and S. K. Joshi, "A survey of optimal power flow methods," J. of Theoretical and App. Info. Tech., vol. 4, no. 5, pp. 450-458, 2008.

[20] M. Kraning, Y. Wang, E. Akuiyibo, and S. Boyd, "Operation and configuration of a storage portfolio via convex optimization," in Proc. of the IFAC World Congress, 2010, pp. 10487-10 492.

[21] P. Denholm and R. Sioshansi, "The value of compressed air energy storage with wind in transmission-constrained electric power systems," Energy Policy, vol. 37, pp. 3149-3158, 2009.

[22] A. E. Sjödin, D. Gayme, and U. Topcu, "Risk-mitigated optimal power flow for wind powered grids," in Proc. of the American Ctrl. Conf., 2012.

[23] S. Bose, F. Gayme, U. Topcu, and K. Chandy, "Optimal placement of energy storage in the grid," in Decision and Control (CDC), 201251 st IEEE Conference on.

[24] J. Lavaei and S. Low, "Zero duality gap in optimal power flow problem," IEEE Trans. on Power Sys., vol. 27, Feb. 2012.

[25] S. Bose, D. Gayme, S. Low, and K. Chandy, "Quadratically constrained quadratic programs on acyclic graphs with application to power flow," To appear in IEEE Transactions on Automatic Control, 2012.

[26] C. Thampoulidis, S. Bose, and B. Hassibi, "Analytic results on the optimal placement of large-scale storage in the grid," 2012, working paper.

[27] IEEE distribution test feeders. [Online]. Available: http://www.ewh.ieee.org/soc/pes/dsacom/testfeeders/index.html

[28] H. Xu, U. Topcu, S. Low, C. Clarke, and K. Chandy, "Load-shedding probabilities with hybrid renewable power generation and energy storage," in Communication, Control, and Computing (Allerton), 2010 48th Annual Allerton Conference on. IEEE, 2010, pp. 233-239.

[29] S. Boyd and L. Vandenberghe, Convex Optimization. Cambridge Univ. Press, 2004 